\title{
On the rate of stimulus occlusion during intermittent retinal stimulation
}

\author{
C. H. BAKER, 1 HUMAN FACTORS RESEARCH, INC. \\ R. A. MONTY, U. S. ARMY HUMAN ENGINEERING LABORATORIES, ABERDEEN PROVING GROUND
}

The CFF was determined with three sectored disks, each of which corresponded to one of the three rings in a Helmholtz disk. The fusion frequency is identical for all three rings of a Helmholtz disk but with the three sectored disks it was found that the rate of stimulus occlusion is a major factor in determining the CFF. It was tentatively concluded that the effect was not a consequence of different stimulus wave-forms. The phenomenon appears to have been reported late in the last century.

In his studies of the duration of the sensation of light, Helmholtz (1911) used a number of rotating disks having different white and black patterns. With respect to one of them, now known as the Helmholtz disk, he states:

Accordingly, if a rotating disk is to give an entirely uniform impression, it must make from 24 to 30 revolutions per second. But the same result can be obtained with slower speeds by repeating the pattern regularly at equal angular intervals. Thus, for instance, in case of the disk in [Fig. 1], it takes six revolutions per second to fuse the black and white of the eight sectors of the outside ring into a uniform grey; while the middle ring takes twelve, and the inside ring takes twenty-four revolutions per second [p. 214].2

A more general statement of the relation was given (in translation) by Schenck and Just (1902): "As we already know, a ring with two sections must for fusing be turned twice as fast as a ring with four alternating black and white sections."

The most recent reference we have found to this phenomenon was made by Zoethout (1947), who asked the following question.

Always assuming that the periodicity is such that the duration of the stimulation is equal to that of the darkness (or black), is the intensity of the uniform sensation affected by the number of stimulations per second? To solve this we may rotate the Helmholtz disk, pictured in [Fig. 1]. With increasing speed it will be seen that the outer border (composed of four white and four black sectors) loses its flicker, first of all, requiring, let us say, but six revolutions per second. The middle ring demands 12 and the inner ring 24 revolutions per second in order to appear as a uniform gray. This is according to what we might have anticipated [p. 194].

In other words, the fusion frequency, as anticipated by Zoethout, is the same for all three rings, viz., 24 stimulations per second.

It has been pointed out by Brown (1965) that most early investigators of the temporal resolution capacity of the eye employed rotating black and white (or colored) disks, but the apparatus now most widely used is a rotating sector disk that interrupts illumination of the test field. With such an apparatus it is easily possible to conduct an experiment that is apparently analogous to that reported by Helmholtz with his disk. Such an experiment is reported here.

\section{Apparatus}

\section{EXPERIMENT 1}

The apparatus consisted of a small enclosed black box with a

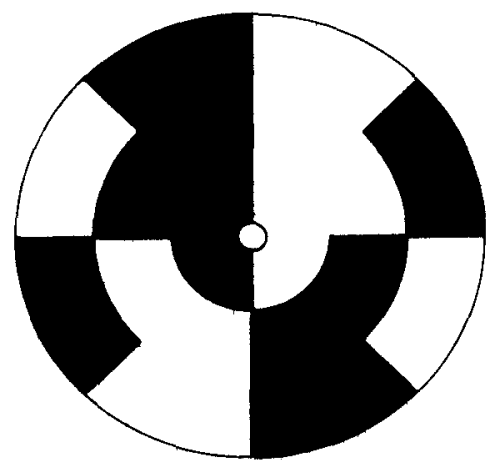

Fig. 1. The Helmholtz diek.

5/8-in. aperture drilled in one side. A $15-W$ bulb, run on dc, was mounted in the box and a sheet of milk glass inside the box covered the aperture. The illuminated aperture, which was viewed from 36 in. and so ensured foveal stimulation, constituted the test field.

A horizontal shaft was mounted under the box, and its speed of rotation was controlled by a variable speed motor. The vanes of a sector disk mounted on the end of the shaft interrupted the light from the test field with any desired frequency. The distance from shaft center to the center of the test field was 2 in. Frequency of interruption was precisely measured by a small light source on one side of the sector disk and a photo-sensitive cell on the other; the output of the cell was fed to an electronic counter.

\section{Method}

The method was simply to determine critical fusion frequencies (CFF) for three sector disks, each of which was identical in design to the three represented in Fig. 1. These three sector disks are shown in Fig. 2. Figure 2a, termed the 180-deg disk, corresponds to the inner-ring pattern in Fig. 1. Figure $2 \mathrm{~b}$, termed the 90-deg disk, corresponds to the middle and Fig. $2 \mathrm{c}$, the 45-deg disk, corresponds to the outer ring of the Helmholtz disk.

Fusion frequencies were recorded in a dimly lit laboratory and the CFF was considered to be the mean value of five sequentially recorded frequencies. Luminance of the test field was such as to produce moderately high values of the CFF. Binocular viewing was employed for all measures. Artificial pupils were not used.
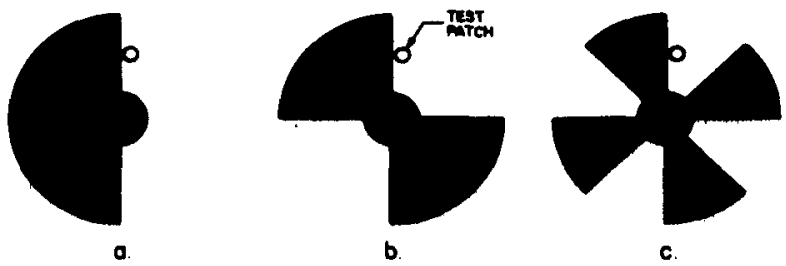

Fig. 2. The three sector disks employed; $2 \mathrm{a}$ is the 180 -deg disk, $2 \mathrm{~b}$ is the 90 -deg disk, and $2 \mathrm{c}$ is the $45-\mathrm{deg}$ disk. 


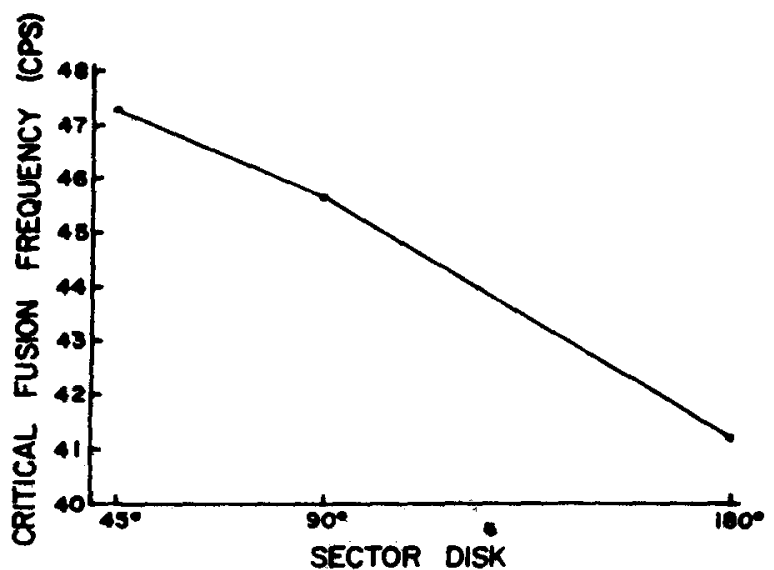

Fig. 3. Mean CFF for each of the three sector disks $(N=21)$.

Subjects were 21 persons between 20 and 30 years of age. Each had 20/20 vision corrected. All Ss were given an hour of training before the data reported here were collected.

\section{Results}

The results in Fig. 3 show that as the sectors increased in angular size the CFF decreased almost linearly. Mean CFF for the 45 -deg disk was $47.3 \mathrm{cps}$, for $90 \mathrm{deg}$ it was $45.7 \mathrm{cps}$, and for $180 \mathrm{deg}$ it was $41.2 \mathrm{cps}$. The differences in CFF between the 45-deg and the 90-deg disks, and between the 90-deg and 180-deg disks are significant $(\mathrm{p}<.01$ and $\mathrm{p}<.001$, respectively).

\section{Discussion}

If this experiment was analogous to that performed with a Helmholtz disk, then CFF would be the same for each of the three disks. But it is not, and an attempt was made to determine the reason for the difference.

When any two sector disks such as $2 \mathrm{a}$ and $2 \mathrm{~b}$ are rotated so as to occlude a test field at the same frequency, e.g., $40 \mathrm{cps}$, then the speed of rotation of $2 \mathrm{a}$ must be double that of $2 \mathrm{~b}$. The single vane of $2 a$, then, occludes and reveals the test field, and the retinal image, in half the time of that taken by a vane of $2 b$. The relation between the speed of disk rotation required to achieve $40 \mathrm{cps}$ (an arbitrary frequency), and the time required by 180 -, 90 - 60, 45-, and $221 / 2$-deg sector disks to occlude a 5/8-in. aperture is shown in Fig. 4. (More precisely, the figure shows the time taken by a point on the edge of a sector vane, 2 in. from the center of rotation, to travel $5 / 8$ in.)

Considered in conjunction with the data in Fig. 3 , it is concluded that there is an inverse relation between rate of occlusion and CFF.

\section{EXPERIMENT 2}

If speed of occlusion of the test field is a major factor in determining the CFF then two test fields of different sizes should result in the same CFF if they are occluded in the same period of time. This was the hypothesis tested in Experiment 2.

\section{Apparatus}

The apparatus was modified so that a $5 / 16$-in. aperture could be easily substituted for the $5 / 8$-in. aperture.

\section{Method}

The method was to determine CFFs for the 5/8-in. aperture, with the 180-deg disk, and for the 5/16-in. aperture with the 90-deg disk. Under these conditions both apertures (one being one-half the diameter and one-quarter the area of the other) were occluded in the same period of time at any given frequency. As a check on the conclusion reached from Experiment 1, CFFs were also determined for the smaller test field, using the f80-deg disk. The same $21 \mathrm{Ss}$ were used.

\section{Results}

The mean CFF for the large aperture and 180-deg disk was virtually identical to that with the small aperture and 90-deg disk, being 41.2 and $41.4 \mathrm{cps}$, respectively. The difference is not statistically significant. In addition, the CFFs for the 90-deg and 180-deg disks, with the smaller aperture, were 41.2 and $38.8 \mathrm{cps}$, respectively, and the difference is significant $(\mathrm{p}<.01)$.

\section{DISCUSSION}

These simple experiments appear to have demonstrated that, for the conditions studied, the rate of stimulus occlusion during intermittent retinal stimulation is a potent factor in determining the CFF-the slower the rate, the greater the CFF.

Figure 5 is a portrayal, to scale, of two conditions of stimulation that were shown to result in different CFFs. The top figure shows, for the 180-deg disk, a $24-\mathrm{msec}$ period from the beginning of an occlusion of the test field to the beginning of the next occlusion. Mean CFF for this condition was found to be $41.2 \mathrm{cps}$. The bottom figure, with a $22 \mathrm{-msec}$ period, is a similar portrayal for the 90-deg disk, with which the mean CFF was found to be $45.7 \mathrm{cps}$.

It is apparent from Fig. 5 that in addition to the rate of occluding (and of revealing) the test field differing in the two conditions, the occluding (and revealing) edges of the disk vanes resulted in a progressive occlusion of the field of luminous energy on the retina of a general sinusoidal nature. ${ }^{3}$ That is, it was sinusoidal with respect to the plane of the retina. However, the sinusoidal occlusion with the 180-deg disk was much more abrupt, much more like a square wave than that with the 90-deg disk. The question arises, then, as to whether or not the results of these experiments are a consequence of differences in stimulus wave-form.

Luckiesh (1914) used a sector disk to study the effect of wave-form upon CFF, that is, "upon the manner in which the illumination varies in passing between the maximum and minimum values." He found that "the more abruptly does the stimulus rise in value the greater the critical frequency." This conclusion, which is quite opposite to that of the experiments reported here, appears to be drawn from a confounded experiment, wave-form being confounded with the light/dark ratio (i.e., the ratio of light and dark in each cycle does not appear to have been constant in all his conditions).

Ives (1922) came to the same general conclusion as Luckiesh

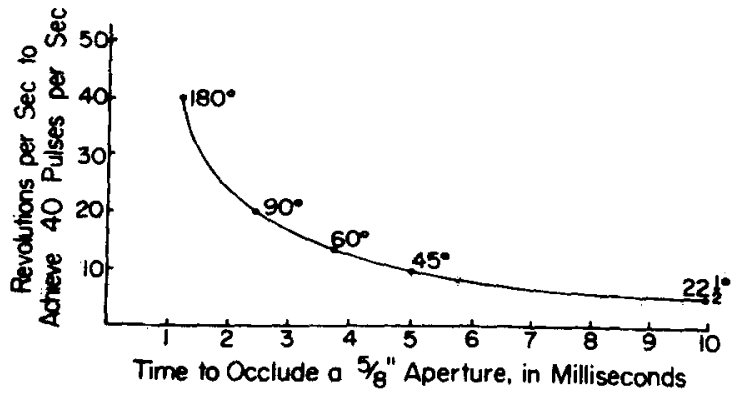

Fig. 4. Showing, for various speeds of rotation, the time to occlude a 5/8-in. aperture located 2 in. from the center of rotation. 

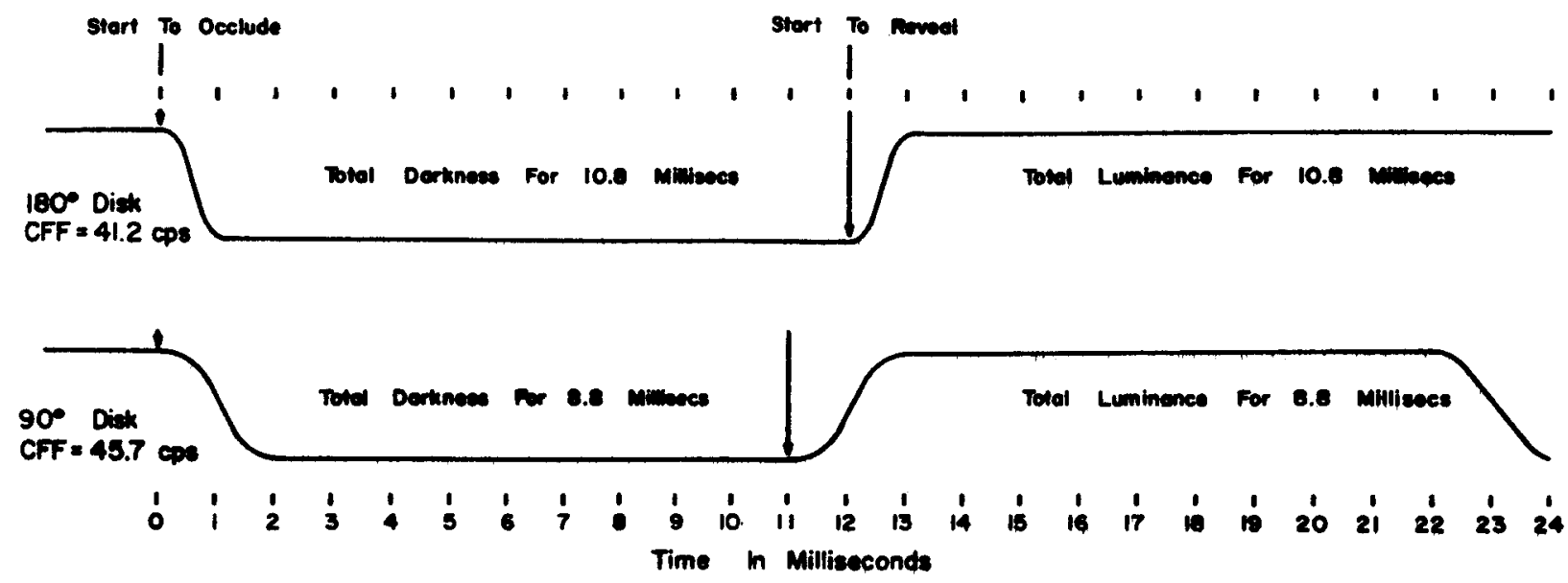

Fiz. 5. Showing a single period, on a time scale, for the 180-deg and 90-deg disks when each was at fusion frequency.

with respect to the effect of wave-form; he found that CFF with a "square-topped" wave was higher by about $2 \mathrm{cps}$ than a "saw-tooth" wave. However, Ives used blue light of low luminance, a condition in which the Ferry-Porter law is not operative. In addition, it is not clear whether or not he held the light/dark ratio constant. Ives' results were examined in detail by Cobb (1934), who claimed that some of them were open to serious question.

Baker and Bott (1951) and Baker (1952) predicted from the alternation of response theory that the CFF of an "instantaneous" light source (that could be turned on and off in 0.00001 sec) would result in a higher CFF than that obtained by progressive occlusion of the source by the vanes of a sector disk. The prediction was supported, the superiority of the CFF obtained with the instantaneous source ranging from 1 to $2 \mathrm{cps}$.

These results, too, are contrary to those reported here. However, Brown (1965) reports a study by Ronchi and Bettini (1957) who found that at low rates of intermittence (below $17 \mathrm{cps}$ ) CFF of a test field that stimulated the rod region occurred at a lower luminance for a saw-tooth stimulus than for a stimulus composed of rectangular pulses. Whether or not this result is contrary to that of Luckiesh, Ives, Baker and Bott, and Baker (and in accord with that reported here), cannot be determined because of differences in experimental conditions, particularly in the retinal region stimulated.

In sum, some of the studies of the effect of wave-form upon the CFF may have generated spurious data, but they are in general agreement: the faster the rate of occlusion the higher the CFF, by from 1 to 2 cps. But in the experiments reported here the relation found was quite the opposite, and the mean difference in CFF (between the 180-deg and 90-deg disks) of $41 / 2 \mathrm{cps}$ is an order of magnitude greater than any of the differences previously reported.

A Fourier analysis was next made of the two wave-forms in Fig. 5 in order to determine whether or not there was any equation of the amplitude of the fundamental components. For this analysis, which had to be done without a computer, the wave-forms were treated as a straight line rather than as the complex wave-forms which they actually are. No equations were found.

It was tentatively concluded that an explanation of the experimental results could not be based upon stimulus wave-form.

The search for an explanation then led to a search of the older literature to determine whether the phenomenon discovered in this study had even been reported before. It had, for rotating black and white disks. Filehne (1885) reported the following (in translation).

It can be demonstrated that the visual organs ... are able to distinguish discontinuous stimulations with rotating disks (at weak or medium daylight) as well as with electric light; only after 75 interruptions per second does the sensation become continuous. When Exner regards 24 intermissions per second as the border line of detectable discontinuity (Plateau gave 60, Aubert gave 50) then the number for Helmholtz's and Emsman's experiments with disks of two to eight sections of black and white are of course correct. But, as one can easily verify, the number of intermissions necessary to produce a continuous effect increases with the number of sections, i.e., decreasing angle of black-white periods, first slowly and then very rapidly. (For brevity we call this value "intermission number"). With 16 and 24 sections the intermission number is about 45 and 60 . From then on it increases more slowly and absolute maximum of 75 is reached with about 48 to 60 sections.

Filehne claimed also that he found the CFF to be the same, $75 \mathrm{cps}$, when he varied "the frequency of electrical illumination of neon light." Rarely, if ever, are CFFs in excess of $60 \mathrm{cps}$ obtained in the laboratory.

Filehne did not attempt an explanation of his results, other than to imply that the reason was obvious (!) by his statement that, "There should not be any need for discussion on why the intermission number never exceeds that maximum [of 75] or becomes smaller than a certain minimum [of 24]." In concluding this reference to Filehne it can be pointed out that he may indeed have been seemg things; his paper is liberally laced with references to his ophthalmological afflictions of "tobacco scotoma" and "flicker scotomas."

The work of a later investigator is also pertinent. Grünbaum (1897, 1898) investigated "to what extent the ratio of the breadth of sector to that of aperture through which observations are made, exerts an influence upon the frequency of intermittence of stimulation at which the sensation of a discontinuous source of light passes into that of a continuous source." Some of Grunbaum's data are reproduced below. It will be noted that the last two CFFs tabled are phenomenally high.

Grübaum offered the following in explanation.

The effect seems to be due to simultaneous contrast which occurs when the field is partly light and partly dark; by increasing the time during which this can take place as in experiment 2 , the sensation of a discontinuous source of 


\section{Table 1}

\begin{tabular}{ccc}
\hline $\begin{array}{c}\text { Alternations to } \\
\text { Produce Fusion }\end{array}$ & $\begin{array}{c}\text { Breadth of } \\
\text { Sector }\end{array}$ & $\begin{array}{c}\text { Diameter of } \\
\text { Aperture }\end{array}$ \\
\hline 43 & 11 & 1 \\
44 & 6.1 & 1 \\
49 & 4.6 & 1 \\
53 & 3.8 & 1 \\
112 & 1.8 & 1 \\
225 & 1 & 1 \\
\hline
\end{tabular}

stimulation persists until the frequency becomes five or six hundred alternations per second. [!]

Using a sector disk (rather than a rotating black and white disk) Grünbaum appears to have discovered the same phenomenon as that reported here, though we view his high CFFs and his explanation with skepticism.

The search for an explanation will continue. In the meantime we have for the Granit-Harper "Law" an interesting exception, viz., two test fields differing in area by a factor of four can have the same CFF.

\section{CONCLUSION}

The rate of stimulus occlusion during intermittent retinal stimulation with a rotating sector disk is a major factor in determining the CFF.

\section{REFERENCES}

BAKER, C. H. The dependence of binocular fusion on timing of peripheral stimuli and on central process. II. Asymmetrical flicker. Canadian Joumal of Psychology, 1952, 6, 123-130.

BAKER, C. H., \& BOTT, E. A. Studies on visual flicker and fusion. II.
Effects of timing of visual stimuli on binocular fusion and flicker Canadian Journal of Psychology, 1951, 5, 9-17.

BROWN. J. L. Flicker and intermittent stimulation. In C. H. Graham (Ed.), Vision and visual perception. New York: Wiley, 1965.

COBB, P. W. Some comments on the Ives theory of flicker. Journal of the Optical Society of America, 1934, 24, 91-98.

FILEHNE, W. Ueber den Entstehungsort des Lichtstaubes, der Stamblindheit und der Nachbilder. V. Graefes Archiv Ophthalmologie 1885,31 (Abt. 2), 1-30.

GRÜNBAUM, O. F. F. On the intermittent stimulation of the retina. I. Journal of Physiology, 1897, 21, 396-402.

GRÜNBAUM, O. F. F. On the intermittent stimulation of the retina. II. Journal of Phy siology, 1898, 22, 433-450.

HELMHOLTZ, H. Physiological optics 3rd. ed., Vol. 2, 1911 (translated by Southall, 1925; Dover edition, 1962).

IVES, H. E. Critical frequency relations in scotopic vision. Journal of the Optical Society of America, 1922, 6, 254-268.

LUCKIESH, M. On the growth and decay of color sensations in flicker photometry. Physical Review, 1914, 4, 1-11.

RONCHI, L., \& BETTINI. On the influence of the shape of equal energy light pulses on the critical flicker frequency. Atti della Fond. Giorgio Ronchi, 1957, 12, 173-179 (briginal not seen).

SCHENCK, F., \& JUST, W. Über intermittierende Netzhautreizung. Pflugers Archiv für gesampte Physiologie, 1902, 90, 270-284.

ZOETHOUT, W. D. Physiological optics 4th ed., Chicago: The Professional Press, 1947.

\section{NOTES}

1. Address: Human Factors Research, Inc., Santa Barbara Research Park, Goleta, California 93017.

2. In the Dover edition Fig. 42 is referred to but the statement is obviously with reference to Fig. 40 . We have not yet been able to determine where, in the series of editions and transiations, this error was initiated.

3. The term sinusoidal is used here as a convenience. Unfortunately the wave-form is much more complex than a sinusoid.

(Accepted for publication, January 10, 1969.) 